OPEN ACCESS

Edited by:

Rosanna Asselta,

Humanitas University, Italy

Reviewed by:

Tohru Yoshihisa,

University of Hyogo, Japan

Rahul N. Kanadia,

University of Connecticut,

United States

${ }^{*}$ Correspondence:

Raymond Kaempfer

kaempfer@hebrew.edu

Specialty section:

This article was submitted to

RNA,

a section of the journal

Frontiers in Genetics

Received: 28 February 2019

Accepted: 30 April 2019

Published: 14 May 2019

Citation:

Kaempfer R, llan L,

Cohen-Chalamish S, Turgeman O,

Namer LS and Osman F (2019)

Control of mRNA Splicing by

Intragenic RNA Activators of Stress

Signaling: Potential Implications

for Human Disease.

Front. Genet. 10:464.

doi: 10.3389/fgene.2019.00464

\section{Control of mRNA Splicing by Intragenic RNA Activators of Stress Signaling: Potential Implications for Human Disease}

\author{
Raymond Kaempfer*, Lena Ilan, Smadar Cohen-Chalamish, Orli Turgeman, \\ Lise Sarah Namer and Farhat Osman
}

Department of Biochemistry and Molecular Biology, The Institute for Medical Research Israel-Canada, The Hebrew University-Hadassah Medical School, Jerusalem, Israel

A critical step in the cellular stress response is transient activation of the RNA-dependent protein kinase PKR by double-helical RNA, resulting in down-regulation of protein synthesis through phosphorylation of the $\alpha$ chain of translation initiation factor elF2, a major PKR substrate. However, intragenic elements of 100-200 nucleotides in length within primary transcripts of cellular genes, exemplified by the tumor necrosis factor (TNF)- $\alpha$ gene and fetal and adult globin genes, are capable of forming RNA structures that potently activate PKR and thereby strongly enhance mRNA splicing efficiency. By inducing nuclear elF2 $\alpha$ phosphorylation, these PKR activator elements enable highly efficient early spliceosome assembly yet do not impair translation of the mature spliced mRNA. The TNF- $\alpha$ RNA activator of PKR folds into a compact pseudoknot that is highly conserved within the phylogeny. Upon excision of $\beta$-globin first intron, the RNA activator of PKR, located in exon 1, is silenced through strand displacement by a short sequence within exon 2 , restricting thereby the ability to activate PKR to the splicing process without impeding subsequent synthesis of $\beta$-globin essential for survival. This activator/silencer mechanism likewise controls splicing of $\alpha$-globin premRNA, but the exonic locations of PKR activator and silencer sequences are reversed, demonstrating evolutionary flexibility. Impaired splicing efficiency may underlie numerous human $\beta$-thalassemia mutations that map to the $\beta$-globin RNA activator of PKR or its silencer. Even where such mutations change the encoded amino acid sequence during subsequent translation, they carry the potential of first impairing PKR-dependent mRNA splicing or shutoff of PKR activation needed for optimal translation.

Keywords: mRNA splicing control, intragenic RNA activators of PKR, activation of PKR, elF2 $\alpha$ phosphorylation, PKR silencer elements, TNF- $\alpha$ gene, $\beta$-globin gene, human $\beta$-thalassemia mutations

Abbreviations: 2-APRE, 2-aminopurine response element; PKR, protein kinase RNA-activated; eIF2 $\alpha$, eukaryotic initiation factor $2 \alpha$-chain; TNF, tumor necrosis factor; IFN, interferon; nt, nucleotide; PBMC, peripheral blood mononuclear cells; UTR, untranslated region. 


\section{INTRODUCTION}

Phosphorylation of the $\alpha$-chain of eukaryotic translation initiation factor 2 (eIF2 $\alpha$ ) is critical for mounting the integrated cellular stress response (Harding et al., 2003; Muaddi et al., 2010). Transient phosphorylation of eIF2 $\alpha$ blocks GDP/GTP exchange needed for recycling of eIF2 between rounds of protein synthesis, inducing translational repression (Sonenberg and Hinnebusch, 2009). The RNA-dependent protein kinase PKR is a prominent eIF2 $\alpha$ kinase having a major role in the IFN-mediated antiviral response. IFNs, including IFN- $\gamma$, induce high levels of $P K R$ gene transcription in the cell (Stark et al., 1998). To become activated, PKR must undergo ATP-dependent trans-autophosphorylation upon engaging, through its tandem RNA binding motifs, double-stranded RNA generated during virus replication (Meurs et al., 1990). Highly ordered doublestranded RNA structures rather than specific sequences are needed to activate PKR (Bevilacqua and Cech, 1996). Once activated by double-stranded RNA, PKR will phosphorylate eIF2 $\alpha$, blocking translation and virus spread from infected cells (Stark et al., 1998).

We review here the discovery and mode of action of a novel class of regulatory RNA elements inside cellular genes that activate PKR to control thereby not only their translation but in particular, enhance their mRNA splicing. Once transcribed into single-stranded RNA, these short non-coding elements fold into structures that act in cis to potently activate PKR, rendering splicing highly efficient (Osman et al., 1999; Ilan et al., 2017; Namer et al., 2017) or repressing translation of the encoded mRNA (Ben-Asouli et al., 2002; Cohen-Chalamish et al., 2009), in each case by inducing eIF2 $\alpha$ phosphorylation. We address potential implications of these RNA elements for human disease.

\section{REGULATION OF GENE EXPRESSION BY INTRAGENIC ELEMENTS THAT ACTIVATE PKR}

Linear double-stranded RNA, generated in the course of virus infection, was considered to be the classical activator of PKR. That notion was shattered by the discovery of short elements within cellular genes that once transcribed, fold into RNA structures capable of activating PKR even more effectively and use this property to control gene expression. Thus, human IFN$\gamma$ mRNA contains a $5^{\prime}$-terminal 203-nt element that folds into a pseudoknot that potently activates PKR, inducing thereby eIF2 $\alpha$ phosphorylation and attenuating its own translation by an order of magnitude (Ben-Asouli et al., 2002; Cohen-Chalamish et al., 2009). This negative feedback loop prevents induction of pathological hyper-inflammation by limiting production of IFN- $\gamma$, a prominent inflammatory cytokine (Ben-Asouli et al., 2002). This intragenic element also couples IFN- $\gamma$ mRNA translation to the level of PKR in the cell (Ben-Asouli et al., 2002). Extensive mutational analysis combined with structure probing showed that the RNA activator of PKR is denatured by ribosome passage and undergoes dynamic refolding to allow
PKR activation in the course of translation (Cohen-Chalamish et al., 2009). Because both activation of PKR and phosphorylation of eIF2 $\alpha$ substrate are transient events, followed promptly by dephosphorylation that inactivates PKR while restoring eIF2 $\alpha$ activity, intragenic RNA activators of PKR function locally as cisacting control elements (Osman et al., 1999; Ben-Asouli et al., 2002; Namer et al., 2017).

\section{TNF- $\alpha$ mRNA SPLICING DEPENDS ON ACTIVATION OF PKR AND PHOSPHORYLATION OF ITS EIF2 $\alpha$ SUBSTRATE}

The inflammatory cytokine TNF- $\alpha$ is not only critical for protective immunity and the anti-tumor response but also a major mediator of inflammatory diseases. TNF- $\alpha$ is expressed promptly during the immune response, TNF- $\alpha$ mRNA levels becoming maximal within $3 \mathrm{~h}$ in stimulated human PBMC (Jarrous et al., 1996). To achieve such efficient expression, splicing of TNF- $\alpha$ mRNA uses activation of PKR. The adenine analog 2-aminopurine, a competitive inhibitor of ATP in binding kinases, especially PKR, blocks splicing of all three TNF- $\alpha$ introns (Jarrous et al., 1996). Splicing of TNF- $\alpha$ pre-mRNA is controlled by the 104-nt 2-APRE located within the $3^{\prime}$-UTR (Figure 1A; Osman et al., 1999). This cis-acting RNA element activates PKR more potently than does double-stranded RNA and enhances TNF- $\alpha$ mRNA splicing by over an order of magnitude when PKR expression is increased (Osman et al., 1999). Mutational analysis, including compensatory mutations that restore base pairing and secondary structure of RNA, showed that the 2-APRE renders nuclear splicing of TNF- $\alpha$ pre-mRNA not only strictly dependent on PKR activation but also highly efficient, yet does not cause translational repression (Osman et al., 1999; Namer et al., 2017). In contrast to TNF- $\alpha$, the closely related TNF- $\beta$ (lymphotoxin) gene does not harbor an intragenic activator of PKR and its mRNA is spliced sluggishly; yet, upon transposition of the TNF- $\alpha$ element into the TNF$\beta 3^{\prime}$-UTR, splicing of TNF- $\beta$ pre-mRNA became as efficient as that of TNF- $\alpha$, showing that the 2-APRE functions as an autonomous splicing control element (Osman et al., 1999; Namer et al., 2017).

Protein kinase RNA-activated activation requires its homodimerization on the activating RNA to permit transautophosphorylation leading to kinase activation (Zhang et al., 2001; Dey et al., 2005). Given that activation of PKR requires at least 33 and optimally 80 base pairs of double-helical RNA (Manche et al., 1992; Bevilacqua and Cech, 1996), how could the 2-APRE, having only $104 \mathrm{nt}$, activate PKR so potently? Extensive genetic analysis, validated by gain-of-function mutations, revealed that the TNF- $\alpha$ RNA activator of PKR folds into a compact pseudoknot that constrains the RNA into two double-helical stacks with parallel axes (Figure 1A), each long enough to bind a PKR monomer, promoting efficient kinase dimerization enabling activation (Namer et al., 2017). The TNF- $\alpha$ pseudoknot is highly conserved in the phylogeny over 400 million years, from teleost fish to humans. Indeed, 

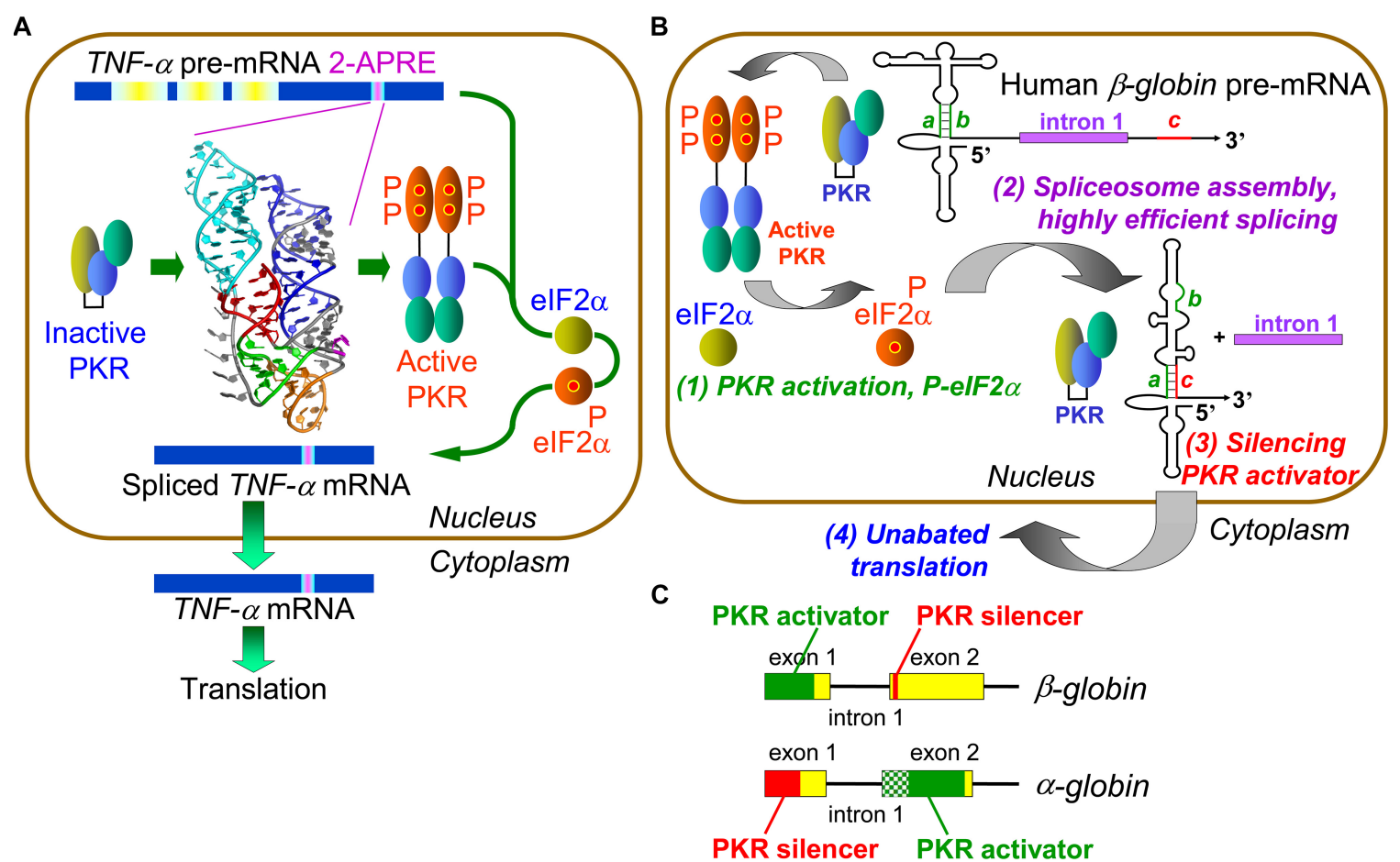

FIGURE 1 | Control of mRNA splicing by intragenic RNA activators of PKR and elF2 $\alpha$ phosphorylation. (A) An RNA element in TNF- $\alpha$ pre-mRNA controls splicing by activating PKR and thereby inducing elF2 $\alpha$ phosphorylation. The 104-nt TNF- $\alpha$ activator of PKR (2-APRE), located within the $3^{\prime}$-UTR, potently activates PKR through its pseudoknot structure that constrains the RNA into two parallel helices, each capable of binding a PKR monomer, facilitating PKR dimerization on RNA needed for kinase activation. Once PKR is activated, it must induce elF-2 $\alpha$ phosphorylation to enable efficient splicing of TNF- $\alpha$ mRNA. (B) Regulation of $\beta$-globin pre-mRNA splicing by an intragenic RNA activator of PKR and silencing of the PKR activator upon intron excision. (1) Within the RNA activator of PKR, consisting of $5^{\prime}$-terminal 124 nt within exon 1, 5 bp helix $a-b$ (green) is critical for activity. Once PKR is activated, it must induce elF-2 $\alpha$ phosphorylation to enable efficient spliceosome assembly and mRNA splicing (2). Upon excision of intron 1, sequence $c$ located near the start of exon 2 (red) displaces strand $b$ from strand $a$, a rearrangement that silences the ability of mature $\beta$-globin mRNA to activate PKR (3). This renders activation of PKR transient, serving solely to promote splicing yet allowing for unimpeded synthesis of $\beta$-globin protein (4) (after Kaempfer et al., 2018). (C) Elements that activate PKR or silence PKR activation map into opposite exons in $\alpha$ - and $\beta$-globin pre-mRNA. The core of the $\alpha$-globin RNA activator of PKR is shown in solid green within exon 2; maximal PKR activation also requires upstream RNA sequence shown in shaded green.

turbot 2-APRE RNA activates human PKR and enhances human TNF- $\beta$ mRNA splicing as effectively as does the human element (Namer et al., 2017).

Local activation of PKR not only enhances TNF- $\alpha$ mRNA splicing but also increases protein yield correspondingly, without repressing translation (Namer et al., 2017). Surprisingly, we discovered that PKR activation promotes efficient TNF- $\alpha$ mRNA splicing by inducing eIF2 $\alpha$ phosphorylation (Figure 1A). Expression of non-phosphorylatable mutant eIF2 $\alpha$ abrogated PKR-dependent splicing. Phosphorylation of eIF2 $\alpha$ is not only strictly needed but also sufficient to achieve highly efficient splicing. Blocking rapid dephosphorylation of eIF $2 \alpha$ with salubrinal, which increases phospho-eIF $2 \alpha$ globally in the cell (Boyce et al., 2005), sufficed to raise splicing the efficiency of TNF- $\beta$ pre-mRNA to that of TNF- $\alpha$ pre-mRNA (Namer et al., 2017). eIF2 $\alpha$ phosphorylation upregulates TNF- $\alpha$ mRNA splicing in human PBMC, demonstrating its physiological relevance. Therefore, stress-induced PKR-mediated eIF $2 \alpha$ phosphorylation has not only a major role in down-regulating translation but also plays a key positive role in rendering splicing highly efficient.

\section{INTRAGENIC RNA ACTIVATORS OF PKR CONTROL GLOBIN GENE EXPRESSION AT MRNA SPLICING}

To analyze the molecular mechanism underlying highly efficient splicing of TNF- $\alpha$ mRNA induced by its intragenic RNA activator of PKR and mediated by eIF $2 \alpha$ phosphorylation, we offered in vitro transcribed $T N F-\alpha$ precursor RNA as substrate for splicing in HeLa cell nuclear extract. That attempt failed, owing to prompt and complete degradation of TNF- $\alpha$ pre-mRNA. However, it led to our discovery that splicing of $\beta$-globin exon 1 intron1-exon2 template, serving as positive control for splicing (Krainer et al., 1984), also depends strictly on the activation of PKR (Ilan et al., 2017). This came as a surprise, given that globin gene expression has long served as a paradigm for eukaryotic gene regulation. Indeed, splicing of human $\alpha$-globin, $\beta$-globin as well as fetal $\gamma$-globin pre-mRNAs depends heavily on PKR activation induced by intragenic RNA activator elements (Ilan et al., 2017). Hence, PKR activation is used more broadly within the human genome beyond inflammatory cytokine genes, to control mRNA 
splicing. Excision of $\beta$-globin intron 1, the first splicing event, was blocked by anti-PKR antibodies as well as by PKR depletion, where it could be restored with recombinant PKR.

The $\beta$-globin RNA activator of PKR maps into the first exon (Figure 1B, step 1); mutation of short helix $a-b$ in the $\beta$-globin activator severely impairs both PKR activation and mRNA splicing. Efficient splicing of each of $\alpha$-, $\beta$ - and $\gamma$-globin premRNA species depends strictly on activation of PKR and nuclear eIF2 $\alpha$ phosphorylation and is inhibited by non-phosphorylatable mutant eIF $2 \alpha$ or anti-phospho-eIF $2 \alpha$ antibodies (Ilan et al., 2017). Activation of PKR and eIF $2 \alpha$ phosphorylation are required at an early step in $\beta$-globin spliceosome assembly, formation of Complex A (Figure 1B, step 2). As shown for $\beta$-globin premRNA, activation of PKR and phosphorylation of eIF $2 \alpha$ mediate splicing not only in vitro but also in intact cells (Ilan et al., 2017).

\section{INTRAGENIC RNA-MEDIATED SILENCING OF PKR ACTIVATORS UPON SPLICING}

The RNA activator of PKR is contained within $\beta$-globin exon 1 and thus maintained in spliced mRNA, where it could strongly down-regulate translation as shown for IFN- $\gamma$ mRNA (Ben-Asouli et al., 2002; Cohen-Chalamish et al., 2009), creating a paradox. Yet, during erythroid development, globin mRNA is translated massively, reaching $95 \%$ of total protein in reticulocytes as compared to $<0.1 \%$ in proerythroblasts (Nienhuis and Benz, 1977). How is maximal translation achieved? Excision of $\beta$-globin first intron juxtaposes short 5 -nucleotide sequence $c$, located near the start of exon 2 , to exon 1 , inducing strand displacement within exon 1 that destroys helix $a-b$ at the core of the PKR activator, resulting in silencing of the activator once $\beta$-globin mRNA is spliced (Figure 1B, step 3 and Figure 1C). Mutation of either strand $a$ or $c$ abrogated silencing whereas compensatory mutation restored it (Ilan et al., 2017). Splicing of $\alpha$-globin pre-mRNA is regulated similarly except that locations of PKR activator and silencer are reversed between exons 2 and 1, demonstrating evolutionary flexibility in control of PKR activation during and upon splicing (Figure 1C). The silencing mechanism allows for highly efficient PKR-dependent splicing, followed promptly by shutoff of PKR activation, to permit undisturbed, maximal translation of the spliced mRNA product (Ilan et al., 2017). This mechanism assures that the ability to activate PKR remains transient, serving only to enable efficient splicing, without hindering globin synthesis (Figure 1B, step 4).

\section{INTRAGENIC RNA ELEMENTS THAT ACTIVATE PKR OR SILENCE PKR ACTIVATORS ARE POTENTIAL SOURCES OF HUMAN DISEASE}

Protein kinase RNA-activated activator and silencer RNA structures were defined for the human $\beta$-globin gene (HBB) by truncation, mutational analysis, and in-line probing of the RNA
(Figure 1B; Ilan et al., 2017). Figure 2 depicts the resulting RNA secondary structure. The activator of PKR is comprised of nucleotides 1-124 from the $5^{\prime}$ end; truncation of only a few nucleotides from either side sufficed to abrogate PKR activation. The AUG start codon, located at positions 51-53, forms part of strand $a$ that generates the helix at the core of the PKR activator. Replacement of CACCA in strand $a$, including A of the start codon, by complementary nucleotides had a pronounced negative effect on splicing efficiency. Replacement of CGUGG in opposite strand $b$, which includes the sequence that engages the AUG codon, by complementary nucleotides largely abrogated splicing efficiency both in cells and in vitro, whereas efficient splicing was restored by $a b$ double mutation (Ilan et al., 2017).

Inspection of the human gene mutation data base $\left(\mathrm{HBB}^{1}\right)$ shows that numerous human $\beta$-thalassemia mutations map to the $\beta$-globin RNA activator of PKR or to its silencer

${ }^{1}$ http://www.hgmd.org

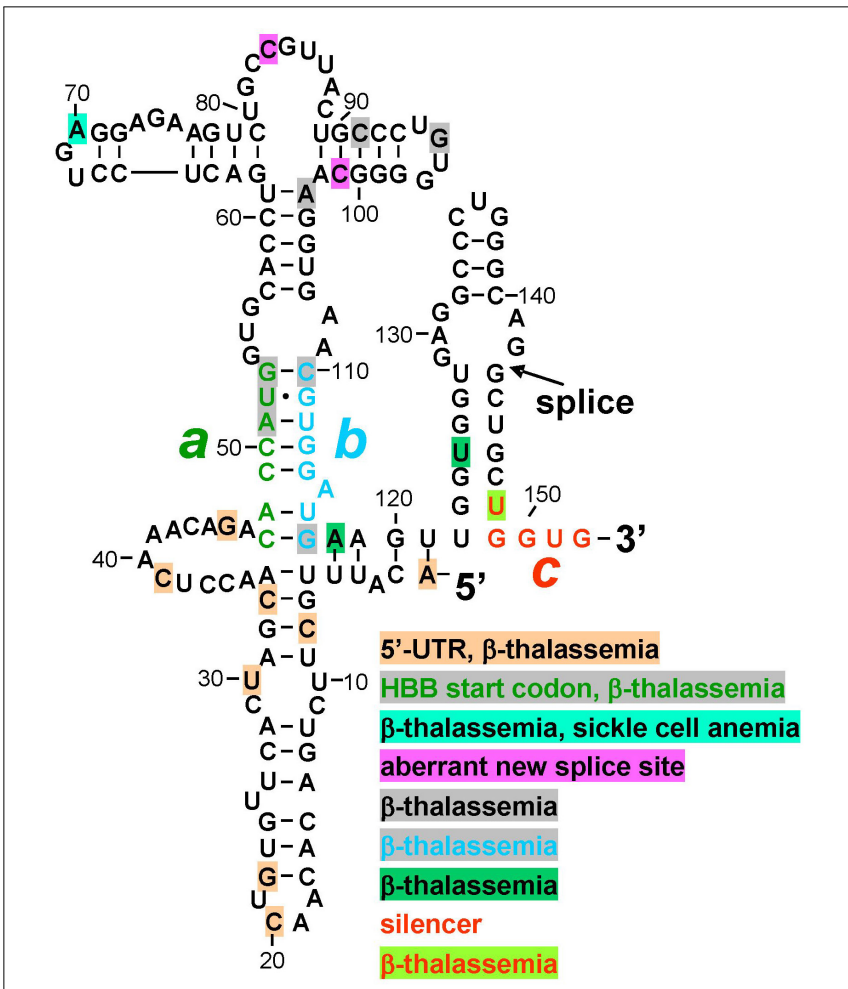

FIGURE 2 | Mutations in human $\beta$-globin RNA activator of PKR and silencer of PKR activation are associated with $\beta$-thalassemia. Structure of the RNA activator of PKR (nucleotides 1-124), determined by in-line probing and mutagenesis, with a key role for helix strands a (green) and $b$ (cyan). Strand $a$ includes the AUG start codon. Position of first splice junction is shown, as is start of exon 2 containing PKR silencer $c$ (red), upon excision of intron 1 but before displacement of strand $b$ by sequence $c$ validated by in-line probing and mutagenesis (see Figure 1B). Nucleotide mutations associated with $\beta$-thalassemia in the human gene mutation database (http://www.hgmd.org), are marked by shading in various colors, see text. HBB, human $\beta$-globin gene. 
(Figure 2). Thus, regulatory mutations were reported within the 5'-UTR, many without mechanism. However, C33G mutation reduced the $\beta$-globin transcript level (Ho et al., 1996) while A1G mutation led, in mouse $\beta$-major globin, to over twofold decreased mRNA expression (Myers et al., 1986); splicing was not analyzed. Other $\beta$-thalassemia mutations map to the AUG initiation codon within strand $a$, to the bifurcation loop structure, to nucleotides in strand $b$ that form G-C base pairs with strand $a$ to generate the helix indispensable for PKR activation, as well as to nucleotides adjoining strand $b$ and the $3^{\prime}$ end of the PKR activator structure (Figure 2). Within silencer sequence $c$, U148A mutation destabilizes base pairing with strand $a$. Because splicing precedes translation, the effect of these mutations may be manifested at the level of PKR activation needed for high splicing efficiency, or at shutoff of PKR activation directly upon splicing, even before they can affect the sequence of the translated $\beta$-globin protein product that is more readily diagnosed in patients and traditionally reported on the data base. Thus, although mutation of the AUG start codon can severely impact translation, this codon also has a dual function in controlling splicing, being located at the heart of the RNA activator of PKR and base pairing with the silencer.

Splicing-defective mutations reported within the $\beta$-globin PKR activator domain (Figure 2) create aberrant splice donor sites that alter protein sequence; aberrant splice donor site mutations are lacking for downstream $\beta$-globin exons 2 and 3.

Minimal sequences encoding the $\alpha$-globin RNA activator of PKR and silencer (Figure 1C) were defined thus far only by truncation analysis (Ilan et al., 2017); their RNA structure remains to be determined. Therefore, it is too early to perform a similar analysis for $\alpha$-globin. Nonetheless, numerous mutations characterized as leading to $\alpha$-thalassemia, hemolytic anemia, or variant $\alpha$-globin proteins (HBA1, HBA2; see footnote 1) map to the PKR activator and silencer domains as delineated at present.

Following the pattern for adult $\beta$-globin, the RNA activator of PKR of $\gamma$-globin, the fetal form of $\beta$-globin, is located within the first exon and $\gamma$-globin mRNA splicing is strictly dependent on PKR activation and eIF2 $\alpha$ phosphorylation (Ilan et al., 2017), but the structure of the $\gamma$-globin PKR activator element was not yet analyzed.

Mutational analysis of the TNF- $\alpha$ RNA activator of PKR (2-APRE, Figure 1A) demonstrated its exquisite sensitivity to mutations, even to a single nucleotide change or base pair inversion, in activating $\mathrm{PKR}$ and rendering splicing highly efficient (Namer et al., 2017). Length and nature of the TNF- $\alpha$ 3'-UTR impart great lability to the primary transcript, rendering detection and analysis of splicing defects in human patients difficult. Assay of TNF- $\alpha$ splicing efficiency necessarily must be done in primary PBMC or transfected cells, using real-time polymerase chain reaction or ribonuclease protection analysis (Namer et al., 2017). It is thus no surprise that among mutations reported as yielding a $T N F-\alpha$ phenotype, the human gene mutation data base lacks thus far mutations mapping into the 2APRE, remote from the coding region. Focus has been on control of TNF- $\alpha$ mRNA translation by microRNAs (TNFA; see footnote 1).

\section{FUTURE PERSPECTIVES}

The discovery of intragenic elements that once transcribed, control splicing by activating PKR in the nucleus or by silencing the ability to activate PKR, adds a new dimension to the analysis and interpretation of human gene mutations. As shown for the RNA activators of PKR within IFN- $\gamma$ mRNA and $T N F-\alpha$ pre-mRNA, even single-nucleotide substitutions or the inversion of a single base pair can lead to loss of the ability of the RNA element to activate PKR (Cohen-Chalamish et al., 2009; Namer et al., 2017). This demonstrates the exquisite sensitivity of the PKR protein molecule to the RNA structure that it must interact with in order to achieve kinase activation, resulting in eIF $2 \alpha$ phosphorylation that in turn enhances mRNA splicing efficiency. As shown for $\beta$-globin pre-mRNA, on the other hand, silencing of the ability of its RNA activator of PKR to activate the kinase, through effective base pairing with RNA encoded by a separate silencer element that induces conformational changes within the RNA activator structure, in this case through strand displacement, is likewise sensitive to mutation (Ilan et al., 2017).

Thus, short intragenic RNA elements that activate PKR or that silence PKR activators are not only essential for controlling efficient mRNA splicing but also create potential etiology for human disease. In a broader sense, this novel perspective may account for and/or contribute to the phenotype of gene mutations analyzed hitherto primarily for their effect on protein sequence. Even where such mutations change the encoded amino acid sequence during subsequent translation in the cytoplasm, they also carry the potential of first impairing PKR-dependent mRNA splicing in the nucleus or the shutoff of PKR activation needed for optimal translation. That concept extends to silent mutations and to mutations that alter amino acid sequence without having a major effect on protein function.

\section{AUTHOR CONTRIBUTIONS}

OT searched the human gene mutation database. OT and RK analyzed the human mutation data. LI, SC-C, LN, FO, and $\mathrm{RK}$ designed and performed the experiments and analyzed the results. RK wrote the manuscript. All authors read and approved the final version of the manuscript for submission.

\section{FUNDING}

This work was supported by United States Congressionally Directed Medical Research Programs award (W81XWH-17-1-0647). 


\section{REFERENCES}

Ben-Asouli, Y., Banai, Y., Pel-Or, Y., Shir, A., and Kaempfer, R. (2002). Human interferon- $\gamma$ mRNA autoregulates its translation through a pseudoknot that activates the interferon-inducible protein kinase PKR. Cell 108, 221-232. doi: 10.1016/s0092-8674(02)00616-5

Bevilacqua, P. C., and Cech, T. R. (1996). Minor-groove recognition of doublestranded RNA by the double-stranded RNA-binding domain from the RNAactivated protein kinase PKR. Biochemistry 35, 9983-9994. doi: 10.1021/ bi9607259

Boyce, M., Bryant, K. F., Jousse, C., Long, K., Harding, H. P., Scheuner, D., et al. (2005). A selective inhibitor of eIF2 $\alpha$ dephosphorylation protects cells from ER stress. Science 307, 935-939. doi: 10.1126/science.1101902

Cohen-Chalamish, S., Hasson, A., Weinberg, D., Namer, L. S., Banai, Y., Osman, F., et al. (2009). Dynamic refolding of IFN- $\gamma$ mRNA enables it to function as PKR activator and translation template. Nat. Chem. Biol. 5, 896-903. doi: 10.1038/nchembio. 234

Dey, M., Cao, C., Dar, A. C., Tamura, T., Ozato, K., Sicheri, F., et al. (2005). Mechanistic link between PKR dimerization, autophosphorylation, and eIF2 $\alpha$ substrate recognition. Cell 122, 901-913. doi: 10.1016/j.cell.2005.06.041

Harding, H. P., Zhang, Y., Zeng, H., Novoa, I., Lu, P. D., Calfon, M., et al. (2003). An integrated stress response regulates amino acid metabolism and resistance to oxidative stress. Mol. Cell 11, 619-633. doi: 10.1016/s1097-2765(03)00105-9

Ho, P. J., Rochette, J., Fisher, C. A., Wonke, B., Jarvis, M. K., Yardumian, A., et al. (1996). Moderate reduction of beta-globin gene transcript by a novel mutation in the $5^{\prime}$ untranslated region: a study of its interaction with other genotypes in two families. Blood 87, 1170-1178.

Ilan, L., Osman, F., Namer, L. S., Eliahu, E., Cohen-Chalamish, S., Ben-Asouli, Y., et al. (2017). PKR activation and eIF2 $\alpha$ phosphorylation mediate human globin mRNA splicing at spliceosome assembly. Cell Res. 27, 688-704. doi: $10.1038 / \mathrm{cr} .2017 .39$

Jarrous, N., Osman, F., and Kaempfer, R. (1996). 2-Aminopurine selectively inhibits splicing of tumor necrosis factor alpha mRNA. Mol. Cell. Biol. 16, 2814-2822. doi: $10.1128 / \mathrm{mcb} \cdot 16.6 .2814$

Kaempfer, R., Namer, L. S., Osman, F., and Ilan, L. (2018). Control of mRNA splicing by noncoding intragenic RNA elements that evoke a cellular stress response. Int. J. Biochem. Cell. Biol. 105, 20-23. doi: 10.1016/j.biocel.2018. 09.021

Krainer, A. R., Maniatis, T., Ruskin, B., and Green, M. R. (1984). Normal and mutant human $\beta$-globin pre-mRNAs are faithfully and efficiently spliced in vitro. Cell 36, 993-1005. doi: 10.1016/0092-8674(84)90049-7

Manche, L., Green, S. R., Schmedt, C., and Mathews, M. B. (1992). Interactions between double-stranded RNA regulators and the protein kinase DAI. Mol. Cell. Biol. 12, 5238-5248. doi: 10.1128/mcb.12.11.5238
Meurs, E., Chong, K., Galabru, J., Thomas, N. S., Kerr, I. M., Williams, B. R., et al. (1990). Molecular cloning and characterization of the human doublestranded RNA-activated protein kinase induced by interferon. Cell 62, 379-390. doi: 10.1016/0092-8674(90)90374-n

Muaddi, H., Majumder, M., Peidis, P., Papadakis, A. I., Holcik, M., Scheuner, D., et al. (2010). Phosphorylation of eIF2 $\alpha$ at serine 51 is an important determinant of cell survival and adaptation to glucose deficiency. Mol. Biol. Cell 21, 3220-3231. doi: 10.1091/mbc.E10-01-0023

Myers, R. M., Tilly, K., and Maniatis, T. (1986). Fine structure genetic analysis of a beta-globin promoter. Science 232, 613-618. doi: 10.1126/science.3457470

Namer, L. S., Osman, F., Banai, Y., Masquida, B., Jung, R., and Kaempfer, R. (2017). An ancient pseudoknot in TNF- $\alpha$ pre-mRNA activates PKR, inducing eIF2 $\alpha$ phosphorylation that potently enhances splicing. Cell Rep. 20, 188-200. doi: 10.1016/j.celrep.2017.06.035

Nienhuis, A. W., and Benz, E. J. (1977). Regulation of hemoglobin synthesis during the development of the red cell. N. Engl. J. Med. 297, 1318-1328. doi: 10.1056/ NEJM197712152972404

Osman, F., Jarrous, N., Ben-Asouli, Y., and Kaempfer, R. (1999). A cis-acting element in the $3^{\prime}$-untranslated region of human TNF- $\alpha$ mRNA renders splicing dependent on the activation of protein kinase PKR. Genes Dev. 13, 3280-3293. doi: $10.1101 / \operatorname{gad} .13 .24 .3280$

Sonenberg, N., and Hinnebusch, A. G. (2009). Regulation of translation initiation in eukaryotes: mechanisms and biological targets. Cell 136, 731-745. doi: 10.1016/j.cell.2009.01.042

Stark, G. R., Kerr, I. M., Williams, B. R., Silverman, R. H., and Schreiber, R. D. (1998). How cells respond to interferons. Annu. Rev. Biochem. 67, 227-264. doi: 10.1146/annurev.biochem.67.1.227

Zhang, F., Romano, P. R., Nagamura-Inoue, T., Tian, B., Dever, T. E., Mathews, M. B., et al. (2001). Binding of double-stranded RNA to protein kinase PKR is required for dimerization and promotes critical autophosphorylation events in the activation loop. J. Biol. Chem. 276, 24946-24958. doi: 10.1074/jbc. M102108200

Conflict of Interest Statement: The authors declare that the research was conducted in the absence of any commercial or financial relationships that could be construed as a potential conflict of interest.

Copyright (C) 2019 Kaempfer, Ilan, Cohen-Chalamish, Turgeman, Namer and Osman. This is an open-access article distributed under the terms of the Creative Commons Attribution License (CC BY). The use, distribution or reproduction in other forums is permitted, provided the original author(s) and the copyright owner(s) are credited and that the original publication in this journal is cited, in accordance with accepted academic practice. No use, distribution or reproduction is permitted which does not comply with these terms. 